\title{
Forkhead box C1 is targeted by microRNA-133b and promotes cell proliferation and migration in osteosarcoma
}

\author{
LU DENG $^{1}$, TANG LIU ${ }^{2}$, BEIBEI ZHANG ${ }^{1}$, HAISHAN WU $^{1}$, JINGPING ZHAO ${ }^{1}$ and JINDONG CHEN ${ }^{1}$ \\ ${ }^{1}$ Mental Health Institute; ${ }^{2}$ Department of Orthopedics, Second Xiangya Hospital of \\ Central South University, Changsha, Hunan 410011, P.R. China
}

Received July 18, 2016; Accepted April 7, 2017

DOI: $10.3892 /$ etm.2017.4870

\begin{abstract}
Forkhead box C1 (FOXC1) has been demonstrated to act as an oncogene in a number of malignant tumors, though its underlying mechanism of action in osteosarcoma (OS) remains unknown. The present study evaluated the expression and regulatory role of FOXC1 in OS. Reverse transcription-quantitative polymerase chain reaction and western blot data indicated that FOXC1 was significantly upregulated in OS tissues and cell lines when compared with adjacent non-tumor tissues $(\mathrm{P}<0.001)$ and normal human osteoblast cells $(\mathrm{P}<0.01)$, respectively. Moreover, levels of FOXC1 expression were significantly higher in OS at advanced clinical stage (III-IV) when compared with that at low clinical stage $(\mathrm{I}-\mathrm{II} ; \mathrm{P}<0.001)$. Knockdown of FOXC1 expression caused a significant decrease in the proliferation and migration of OS U2OS cells $(\mathrm{P}<0.01)$, while overexpression of FOXC1 significantly promoted U2OS cell proliferation and migration $(\mathrm{P}<0.01)$, relative to control U2OS cells. Furthermore, FOXC1 was identified as a direct target of microRNA (miR)-133b, a reported tumor-suppressive miR in OS. The protein expression of FOXC1 was negatively regulated by miR-133b in U2OS cells $(\mathrm{P}<0.01)$, and miR-133b expression was inversely correlated with $\mathrm{FOXC1}$ expression in OS. In conclusion, the present study demonstrated that FOXC1, targeted by miR-133b, may promote cell proliferation and migration in OS. Thus, FOXC1 may be a potential therapeutic target in the treatment of OS.
\end{abstract}

\section{Introduction}

Osteosarcoma (OS) is a common malignant primary tumor of the bone, which primarily affects children and adolescents $(1,2)$.

Correspondence to: Professor Jindong Chen, Mental Health Institute, Second Xiangya Hospital of Central South University, 139 Middle Renmin Road, Changsha, Hunan 410011, P.R. China E-mail: xychenjindong@sina.com

Key words: osteosarcoma, forkhead box C1, oncogene, microRNA
The standard treatment for OS consists of surgery and intensive multi-agent chemotherapy, which has improved the 5-year survival rate of patients with OS. However, 30-40\% of OS patients present with pulmonary metastasis and relapse, and have a poor prognosis $(2,3)$. In addition, the cellular and molecular mechanisms underlying the growth and metastasis of OS remain unknown.

Forkhead box C1 (FOXC1), as a member of the FOX protein family, is a transcription factor containing a characteristic DNA-binding forkhead domain (4). Previous studies have documented that FOXC1 takes part in the regulation of embryonic and ocular development $(4,5)$. More recently, it has been observed that the regulation of FOXC1 is perturbed in a number of human cancers, including sarcomas $(6,7)$. In basal-like breast cancer (BLBC), FOXC1 was significantly upregulated, and its upregulation was associated with poor prognosis of patients with BLBC (8). Moreover, knockdown of FOXC1 significantly reduced the proliferation, migration and invasion of BLBC cells (8). FOXC1 may also contribute to microvascular invasion in hepatocellular carcinoma by promoting epithelial-mesenchymal transition (EMT) (9), and in synovial sarcoma, upregulation of FOXC1 has been observed (10). However, the expression and clinical significance of FOXC1, and the potential benefits of targeting FOXC1 as a putative therapeutic strategy for OS are unknown.

MicroRNAs (miRs), as a type of short non-coding RNAs, inhibit the protein expression of their target genes by binding to the 3'-untranslated region (UTR) of mRNAs, which leads to the inhibition of protein translation and RNA degradation (11). By regulating the expression of their target genes, miRs may affect cell proliferation, differentiation, apoptosis, migration and tumorigenesis $(12,13)$. Recently, a number of miRs have been demonstrated to serve suppressive roles in different human cancers through the inhibition of FOXC1 expression (14-16). For instance, in nasopharyngeal carcinoma, miR-4792 inhibited EMT and the invasion of carcinoma cells through the repression of FOXC1 expression (15). In endometrial cancer, MiR-495 suppressed the growth and migration of cancer cells by targeting FOXC1 (16). However, there is a lack of studies into the epigenetic regulatory mechanism underlying the expression of FOXC1 in OS.

In the present study, the expression and regulatory role of FOXC1 was evaluated in human OS. In addition, the epigenetic 
regulatory mechanism underlying the expression of $\mathrm{FOXC1}$ in human OS cells was investigated.

\section{Materials and methods}

Tissue collection. The current study was approved by the Ethics Committee of the Second Xiangya Hospital of Central South University (Changsha, China). Written informed consent was obtained from all patients in the present study. A total of 42 primary OS tissues and matched adjacent non-tumor tissues were collected at the Second Xiangya Hospital of Central South University from March 2012 to May 2014. The tissue samples were immediately snap-frozen in liquid nitrogen following surgical resection and stored at $-80^{\circ} \mathrm{C}$ until use. The clinical characteristics of patients involved in the current study are presented in Table I. TNM I-II stage was recognized as low stage and TNM III-IV stage was recognized as high stage (17). Patients were diagnosed at the Pathology Department of the Second Xiangya Hospital of Central South University. No chemotherapy or radiotherapy was administered prior to surgical resection.

Reverse transcription-quantitative polymerase chain reaction $(R T-q P C R)$. Total RNA was extracted from patient tissues using TRIzol ${ }^{\circledR}$ reagent (Thermo Fisher Scientific, Inc., Waltham, MA, USA) and converted into cDNA using a RevertAid Reverse Transcription kit (Thermo Fisher Scientific, Inc.), according to the manufacturer's instructions. For mRNA detection, a SYBR-Green I Real-Time PCR kit (Biomics Biotechnologies Co., Ltd., Nantong, China) was used to perform real-time qPCR. GAPDH was used as an internal control. The primer sequences used were as follows: For FOXC1 forward, 5'-GGCGAGCAGAGCTACTACC-3' and reverse, 5'-TGCGAGTACACGCTCATGG-3' and for GAPDH forward, 5'-ACAACTTTGGTATCGTGGAAGG-3' and reverse, 5'-GCCATCACGCCACAGTTTC-3'. For miR detection, an All-In-One miRNA qRT-PCR Detection kit (GeneCopoeia, Inc., Rockville, MD, USA) was used to conduct real-time qPCR, according to the manufacturer's instructions. The reaction conditions for both mRNA and miRNA PCR were $95^{\circ} \mathrm{C}$ for $5 \mathrm{~min}$, followed by 40 cycles of denaturation at $95^{\circ} \mathrm{C}$ for $15 \mathrm{sec}$ and an annealing/elongation step at $60^{\circ} \mathrm{C}$ for $30 \mathrm{sec}$. Relative expression was analyzed using the $\Delta \Delta \mathrm{Cq}$ method (18). GAPDH was used for mRNA normalization and U6 for miR normalization. Three replicates were performed for each experiment.

Cell culture. The human OS cell lines Saos-2, MG63, U2OS and KHOS, and normal human osteoblast hFoB1.19 cells were purchased from the Cell Bank of Type Culture Collection of Chinese Academy of Sciences (Shanghai, China). The cell lines were cultured in RPMI-1640 medium (Invitrogen; Thermo Fisher Scientific, Inc.) supplemented with $10 \%$ fetal bovine serum (FBS), $100 \mathrm{U} / \mathrm{ml}$ penicillin and $100 \mu \mathrm{g} / \mathrm{ml}$ streptomycin (all from Thermo Fisher Scientific, Inc.) at $37^{\circ} \mathrm{C}$ in a $5 \% \mathrm{CO}_{2}-95 \%$ air atmosphere.

Bioinformatics prediction. TargetScan software (http://www. targetscan.org) was used to predict the potential miRs that target FOXC1. 'Human' was selected as the species, and
Table I. Clinical characteristics of patients with osteosarcoma.

\begin{tabular}{|c|c|}
\hline Variable & Osteosarcoma \\
\hline Number of patients, $n$ & 42 \\
\hline Age range (mean, years) & $16-48(31.5 \pm 10.2)$ \\
\hline \multicolumn{2}{|l|}{ Sex } \\
\hline Male & 25 \\
\hline Female & 17 \\
\hline \multicolumn{2}{|l|}{ Clinical stage } \\
\hline I & 7 \\
\hline II & 15 \\
\hline III & 14 \\
\hline IV & 6 \\
\hline
\end{tabular}

'FOXC1' was entered. miR-133b was identified and selected as the potential target gene of FOXC1.

Cell transfection. Cells were transfected with $100 \mathrm{nM}$ of FOXC1 small interfering (si)-RNA, non-specific siRNA [negative control (NC) siRNA], pcDNA3.1-FOXC1 expression plasmid, pcDNA3.1 vector, miR-133b mimic, scramble miR (miR-NC), miR-133b inhibitor or NC inhibitor (all purchased from Yearthbio, Changsha, China) using Lipofectamine ${ }^{\circledR} 2000$ (Thermo Fisher Scientific, Inc.), according to the manufacturer's instructions.

Western blot analysis. Cells were lysed with ice-cold lysis buffer (Beyotime Institute of Biotechnology, Haimen, China) and centrifugation was performed at $12,000 \mathrm{x}$ g at $4^{\circ} \mathrm{C}$ for $10 \mathrm{~min}$. Protein concentration was determined using a bicinchoninic acid protein acid kit (Santa Cruz Biotechnology, Inc., Dallas, TX, USA). Proteins (60 $\mu \mathrm{g})$ were separated by $10 \%$ SDS-PAGE (Beyotime Institute of Biotechnology) and transferred onto a polyvinylidene difluoride (PVDF) membrane (Thermo Fisher Scientific, Inc.). The PVDF membrane was incubated with phosphate-buffered saline (PBS) containing 5\% non-fat milk (Mengniu Dairy Co., Ltd., Hong Kong, China) overnight at $4^{\circ} \mathrm{C}$, followed by incubation with rabbit anti-human FOXC1 antibody (1:50, ab24067) and GAPDH antibodies (1:100, ab9485) (both from Abcam, Cambridge, MA, USA) at room temperature for 3 h. Following 3 washes with PBS with Tween-20, the membrane was incubated with horseradish peroxidase-conjugated goat anti-rabbit secondary antibodies (1:5,000, ab97051; Abcam) at room temperature for $1 \mathrm{~h}$. An enhanced chemiluminescence system (Thermo Fisher Scientific, Inc.) was used to detect the immunoreactive bands. Protein expression was measured using Image-Pro Plus 6.0 software (Media Cybernetics, Inc., Rockville, MD, USA). GAPDH was used as an internal reference. Three replicates were performed.

MTT assay. Suspended U2OS cells were plated into 96-well plates $\left(5 \times 10^{4}\right.$ cells/well) and cultured for $0,24,48$ or $72 \mathrm{~h}$ in RPMI-1640 medium with $10 \% \mathrm{FBS}$ at $37^{\circ} \mathrm{C}$. The viability of U2OS cells was determined using an MTT assay. Briefly, following cell culture, $20 \mu \mathrm{l} \mathrm{MTT}(5 \mathrm{mg} / \mathrm{ml}$; Sigma-Aldrich; Merck KGaA, Darmstadt, Germany) was added to each well 


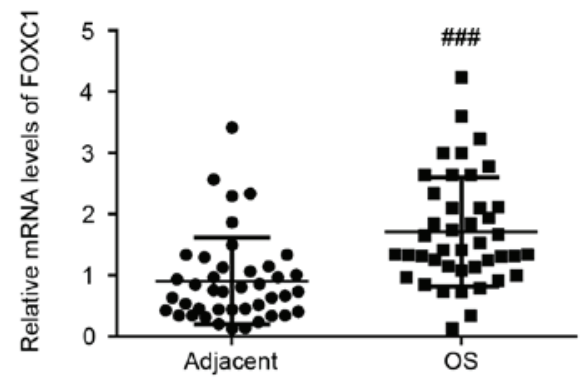

C

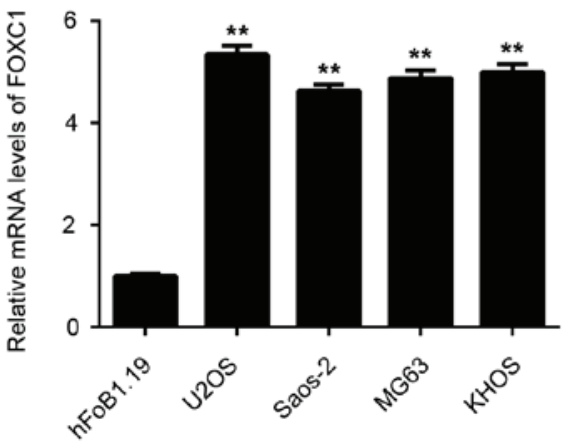

B

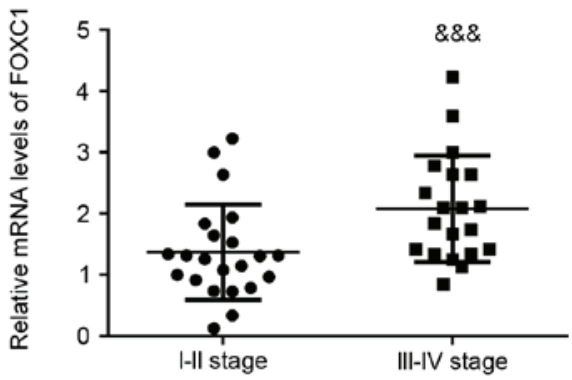

D
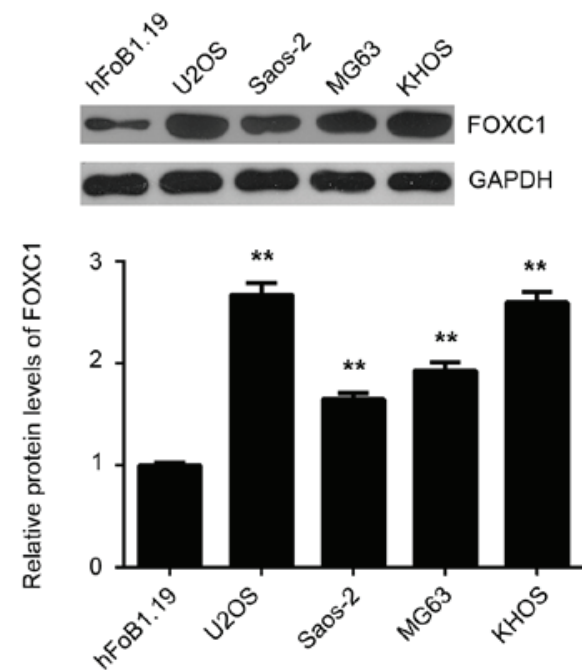

Figure 1. Upregulation of FOXC1 in OS. (A) RT-qPCR was used to measure the levels of FOXC1 mRNA in OS tissues and adjacent non-tumor tissues. (B) RT-qPCR was used to assess the levels of FOXC1 mRNA in OS tissues at different clinical stages. (C) RT-qPCR and (D) western blot analysis were performed to measure the expression of FOXC1 at the mRNA and protein levels in OS cell lines and normal osteoblast hFoB1.19 cells. ${ }^{\# \# ~} \mathrm{P}<0.001 \mathrm{vs}$. adjacent tissue; \&\&\& $<0.001$ vs. I-II stage OS. ${ }^{* *} \mathrm{P}<0.01$ vs. $\mathrm{hFoB} 1.19$ cells. FOXC1, forkhead box C1; OS, osteosarcoma; RT-qPCR, reverse transcription-quantitative polymerase chain reaction.

and the 96 -well plates were incubated at $37^{\circ} \mathrm{C}$ in a humidified $5 \% \mathrm{CO}_{2}$ atmosphere for $4 \mathrm{~h}$. The resulting formazan product was dissolved with $100 \mu \mathrm{l}$ isopropanol and absorbance at $490 \mathrm{~nm}$ was measured with a SpectraMax Microplate ${ }^{\circledR}$ Spectrophotometer (Molecular Devices LLC, Sunnyvale, CA, USA).

Wound-healing assay. U2OS cells (control, FOXC1 siRNA and FOXCI groups) were cultured in a 6-well plate in RPMI-1640 medium containing $10 \% \mathrm{FBS}$ at $37^{\circ} \mathrm{C}$ (initial cell density, $1 \times 10^{5}$ cells/well) into $100 \%$ confluence, and a wound was created in the cell monolayer with a plastic scriber. Cells were washed and cultured in RPMI-1640 containing 10\% FBS at $37^{\circ} \mathrm{C}$ for $48 \mathrm{~h}$. The wounds were then photographed under a Nikon microscope (Instruments, Inc., Sterling Heights, MI, USA) equipped with Zen Imaging 1.0 software (Zeiss AG, Oberkochen, Germany). The wound width was evaluated by measuring the distance between the two edges of the scratch at 5 random sites in each image.

Luciferase reporter assay. A mutant type (MT) of the FOXC1 3'UTR lacking complementarity with the miR-133b seed sequence was generated using a QuickChange Site-Directed Mutagenesis kit (Stratagene; Agilent Technologies, Inc., Santa Clara, CA, USA), according to the manufacturer's instructions. The wild-type (WT) or MT FOXC1 3'UTR was cloned into a
pMIR-GLO ${ }^{\mathrm{TM}}$ Luciferase vector (performed by Yearthbio) downstream of the firefly luciferase coding region. U2OS cells were co-transfected with $100 \mathrm{nM}$ WT-FOXC1-3'UTR or MT-FOXC1-3'UTR plasmid and $100 \mathrm{nM}$ miR-NC or miR-133b mimic using Lipofectamine 2000. Luciferase activity was detected $48 \mathrm{~h}$ after transfection using a Dual-Luciferase ${ }^{\circledR}$ Reporter Assay system (Promega Corporation), according to the manufacturer's instructions.

Statistical analysis. Data were expressed as the mean \pm standard deviation of three independent experiments. SPSS 19.0 software (IBM Corp., Armonk, NY, USA) was used for statistical analysis. Spearman's rank correlation analysis was used to examine the relationship between miR-133b and FOXC1 expression in OS tissues. Significance differences between groups were analyzed using a Student's t-test or one-way analysis of variance with Tukey's post hoc test, and $\mathrm{P}<0.05$ was considered to indicate a statistically significant difference.

\section{Results}

Upregulation of FOXC1 in OS tissues and cell lines is associated with tumor progression. In the present study, RT-qPCR and western blot analysis were conducted to assess the mRNA and protein expression of FOXC1 in OS tissues and cell lines. 
A

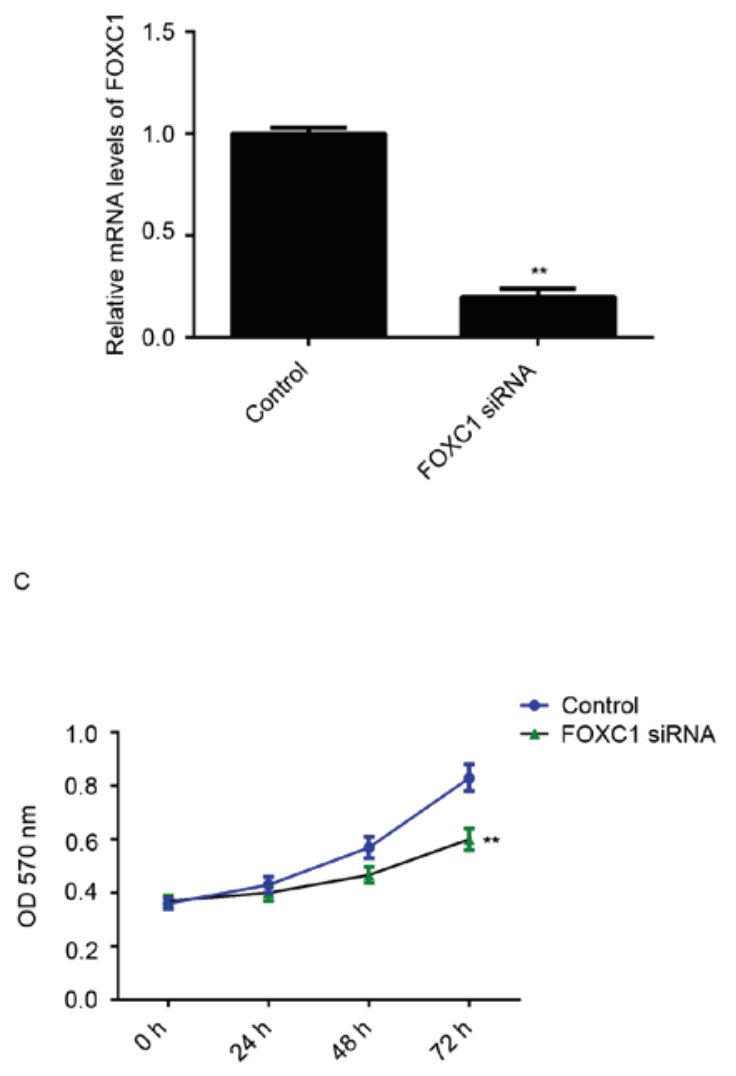

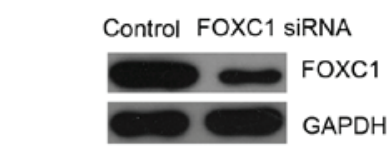
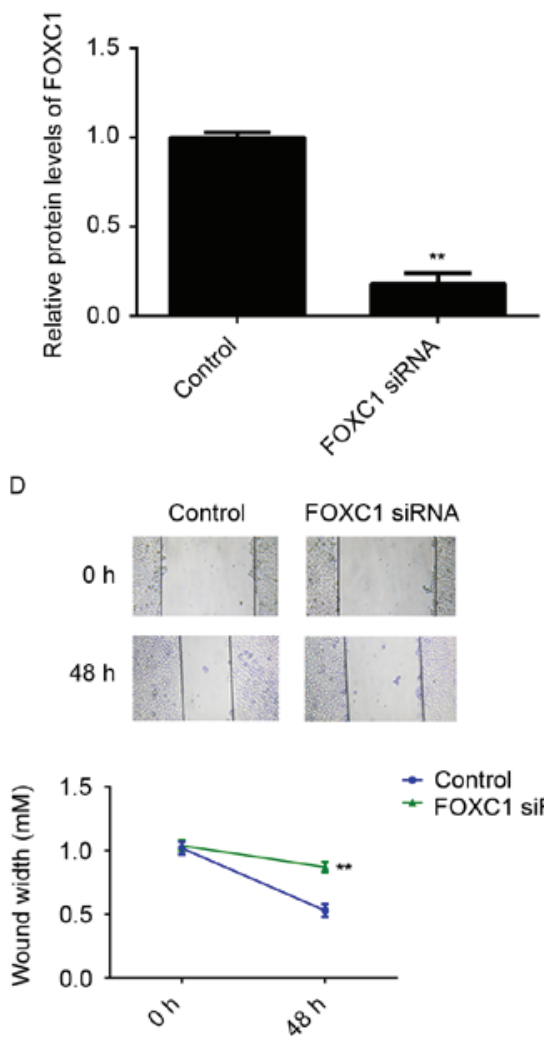

- Control

- FOXC1 SiRNA

Figure 2. Effects of FOXC1 knockdown on OS cell proliferation and migration. The OS cell line U2OS was transfected with FOXC1 siRNA or with non-specific siRNA, as a control. (A) Reverse transcription-quantitative polymerase chain reaction and (B) western blot analysis were performed to measure the expression of FOXC1 at the mRNA and protein levels. (C) MTT and (D) wound healing assays were conducted to assess the proliferation and migration of transfected cells. Magnification, $\mathrm{x} 40 .{ }^{* *} \mathrm{P}<0.01$ vs. control. FOXC1, forkhead box C1; OS, osteosarcoma; siRNA, small interfering RNA.

Adjacent non-tumor tissues and normal human osteoblast hFoB1.19 cells were used as controls, respectively. As depicted in Fig. 1A, FOXC1 mRNA was significantly upregulated in OS tissues compared with adjacent non-tumor tissues $(\mathrm{P}<0.001)$. In addition, the expression of FOXC1 mRNA was significantly higher in high stage OS (III-IV) when compared with that in low stage OS (I-II; $\mathrm{P}<0.001$; Fig. 1B), suggesting that increased expression of FOXC1 may contribute to the malignant progression of OS. RT-qPCR and western blot data also indicted that FOXC1 expression was significantly increased in the OS cell lines Saos-2, MG63, U2OS and KHOS, when compared with that in normal human osteoblast hFoB1.19 cells $(\mathrm{P}<0.01$; Fig. 1C and D). Collectively, these findings indicate that FOXC1 is upregulated in OS.

FOXC1 promotes OS cell proliferation and migration. As FOXC1 was significantly upregulated in OS, U2OS cells were transfected with FOXC1 siRNA to decrease its expression. Transfection with non-specific siRNA was used as a control. As indicated in Fig. 2A and B, transfection with FOXC1 siRNA caused a significant reduction in FOXC1 at the mRNA and protein levels, relative to the control group $(\mathrm{P}<0.01)$. MTT and wound healing assays were subsequently performed to evaluate cell proliferation and migration. It was observed that knockdown of FOXC1 significantly decreased the proliferation and migration of U2OS cells compared with the control group $(\mathrm{P}<0.01$; Fig. 2C and D). To verify these findings, U2OS cells were transfected with FOXC1 mimic to upregulate its expression. Transfection with miR-NC was used as a control. RT-qPCR and western blot data indicated that FOXC1 expression was significantly higher in the FOXC1 group compared with the control group $(\mathrm{P}<0.01$; Fig. $3 \mathrm{~A}$ and $\mathrm{B})$. Moreover, overexpression of FOXC1 promoted the proliferation and migration of U2OS cells $(\mathrm{P}<0.01$ vs. control; Fig. $3 \mathrm{C}$ and $\mathrm{D})$. These data indicate that $\mathrm{FOXC1}$ may promote the proliferation and migration of OS cells.

FOXC1 is a target gene of miR-133b in OS cells. The epigenetic regulatory mechanism underlying the expression of FOXC1 in OS was investigated. Targetscan software was used to analyze the potential miRs that target FOXC1. As depicted in Fig. 4A and B, FOXC1 was predicted to be a target gene of miR-133b, which is considered to be a tumor suppressor in OS (19). To verify this targeting relationship, WT or MT FOXC1 3'UTR was cloned into a pMIR-GLO ${ }^{\mathrm{TM}}$ Luciferase vector downstream of the firefly luciferase coding region, generating WT- or MT-FOXC1-3'UTR luciferase reporter plasmids, respectively (Fig. 4C and D). U2OS cells were then co-transfected with WT-FOXC1-3'UTR or MT-FOXC1-3'UTR plasmid and miR-NC or miR-133b 
A

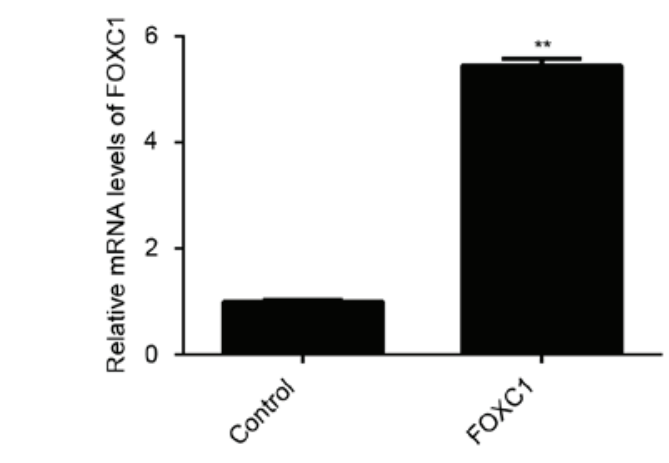

C

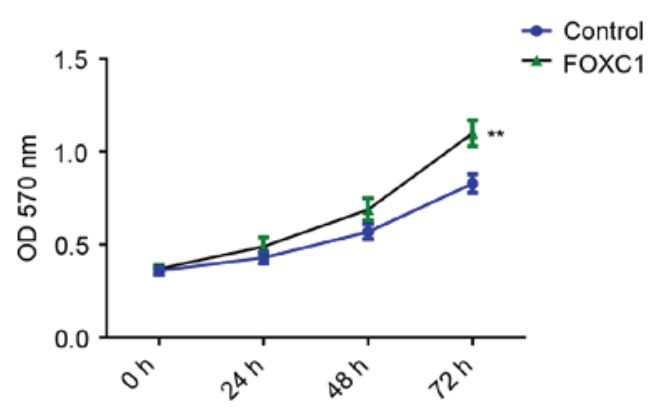

B

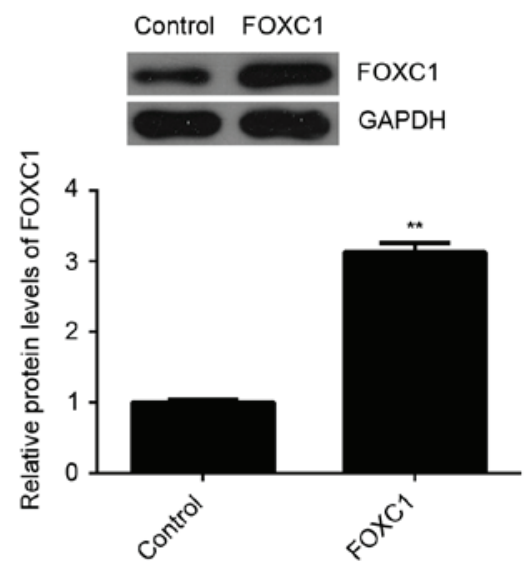

D
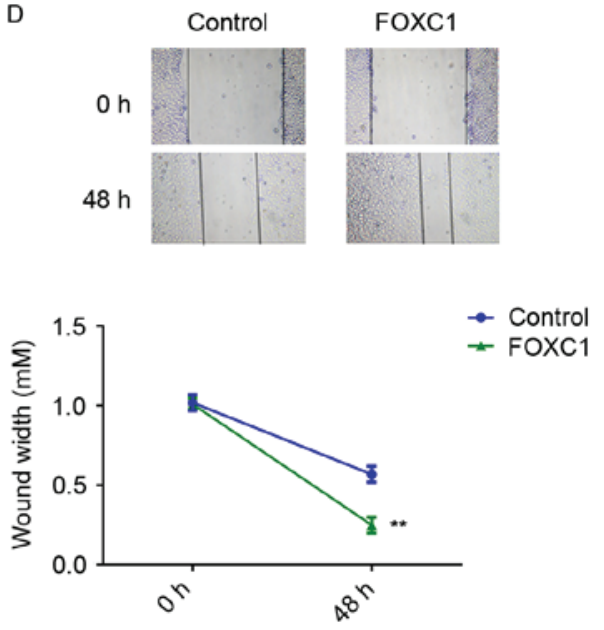

Figure 3. Effects of FOXC1 overexpression on OS cell proliferation and migration. The OS cell line U2OS was transfected with a pcDNA3.1-FOXC1 expression plasmid or with a blank vector, as a control. (A) Reverse transcription-quantitative polymerase chain reaction and (B) western blot analysis were performed to measure the expression of FOXC1 at the mRNA and protein levels. (C) MTT and (D) wound healing assays were conducted to assess the proliferation and migration of transfected cells. Magnification, $\mathrm{x} 40 .{ }^{* *} \mathrm{P}<0.01$ vs. control. FOXC1, forkhead box C1; OS, osteosarcoma.

mimic. As depicted in Fig. 4E, luciferase activity was significantly decreased in U2OS cells co-transfected with miR-133b mimic and WT-FOXC1-3'UTR plasmid ( $\mathrm{P}<0.01$ vs. miR-NC + WT-FOXC1-3'UTR cells). By contrast, luciferase activity was unaffected in cells co-transfected with miR-133b mimic and MT-FOXC1-3'UTR plasmid when compared with miR-NC + MT-FOXC1-3'UTR cells. These data indicate that miR-133b may directly bind to the 3'UTR of FOXC1 mRNA in U2OS cells.

The effect of miR-133b on the expression of FOXC1 was further evaluated in U2OS cells. U2OS cells were transfected with miR-133b mimic or miR-NC, as a control. Following transfection, levels of miR-133b were significantly increased in the miR-133b group compared with the control group $(\mathrm{P}<0.01$; Fig. 5A). In turn, western blot data indicated that overexpression of miR-133b led to a significant decrease in the levels of FOXC1 protein in U2OS cells $(\mathrm{P}<0.01$; Fig. 5B). To verify these findings, U2OS cells were transfected with miR-133b inhibitor or NC inhibitor, as a control. Transfection with miR-133b inhibitor caused a significant reduction in the levels of miR-133b when compared with the control group $(\mathrm{P}<0.01$; Fig. 5C). In turn, knockdown of miR-133b significantly increased the expression of FOXC1 protein in U2OS cells
$(\mathrm{P}<0.01$; Fig. 5D). Therefore, miR-133b may negatively regulate the expression of $\mathrm{FOXC1}$ protein in OS cells.

miR-133b expression is inversely correlated with FOXC1 expression in $O S$. To verify the relationship between miR-133b and FOXC1 in OS, the expression of miR-133b was assessed in OS tissues and cell lines using RT-qPCR. It was observed that levels of miR-133b were significantly reduced in OS tissues compared with adjacent non-tumor tissues ( $\mathrm{P}<0.001$; Fig. 6A). Similarly, miR-133b was downregulated in OS cell lines when compared with normal osteoblast hFoB1.19 cells ( $\mathrm{P}<0.01$; Fig. 6B). Moreover, levels of miR-133b were inversely correlated with levels of FOXC1 in OS tissues ( $R=-0.64, \mathrm{P}<0.01$; Fig. 6C), suggesting that a downregulation of miR-133b may contribute to the upregulation of FOXC1 in OS.

\section{Discussion}

There is a lack of studies into the exact roles of FOXC1 in the growth and metastasis of OS. In the present study, it was observed that FOXC1 was significantly upregulated in OS tissues and cell lines when compared with adjacent non-tumor tissues and normal human osteoblast cells, respectively. 
A

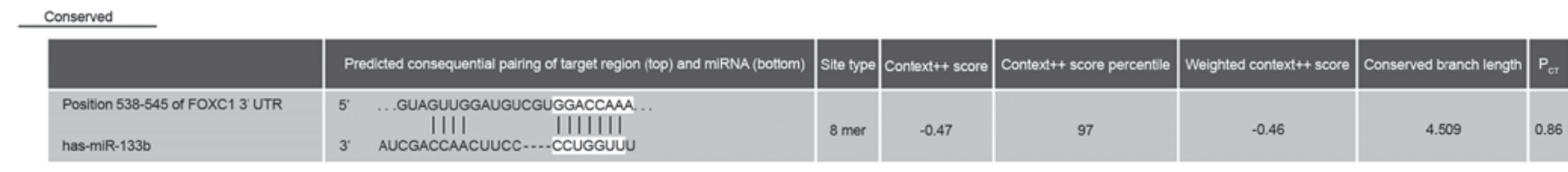

B

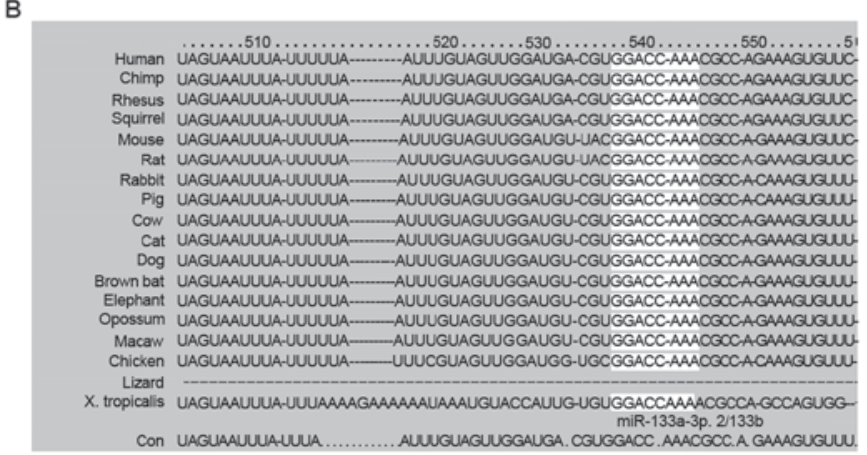

D
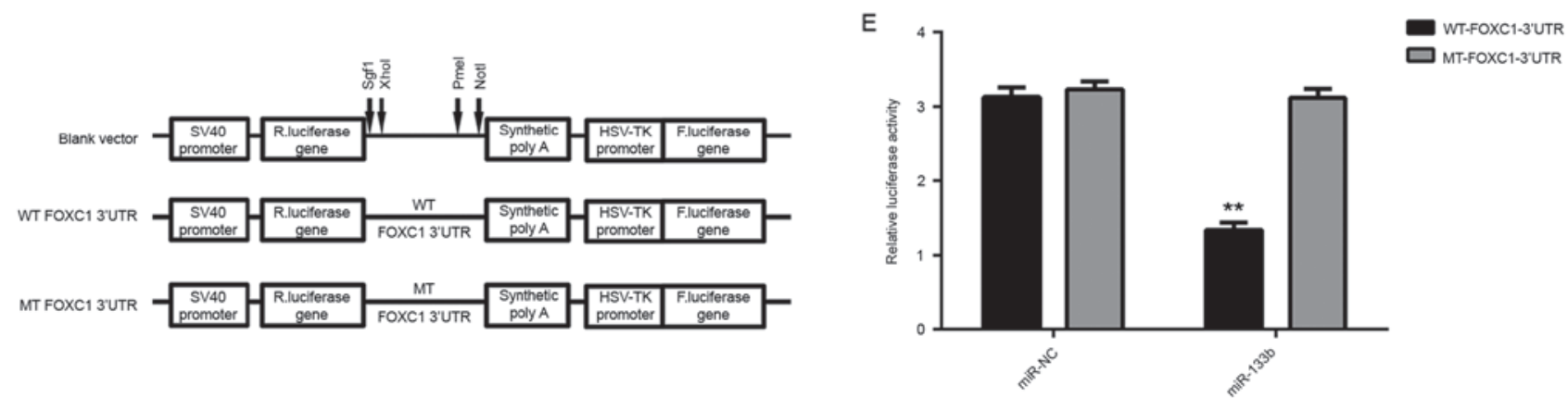

Figure 4. miR-133b targets FOXC1 in OS. (A) Targetscan software predicted that FOXC1 was a putative target gene of miR-133b, and that (B) their targeting relationship was evolutionally conserved. (C and D) WT- or MT-FOXC1 3'UTR was cloned into a pMIR-GLOTM Luciferase vector downstream of the F. luciferase coding region. (E) Luciferase activity was significantly decreased in U2OS cells co-transfected with miR-133b mimic and WT-FOXC1-3'UTR plasmid but not in cells co-transfected with miR-133b mimic and MT-FOXC1-3'UTR plasmid. "** $\mathrm{P}<0.01$ vs. miR-NC. FOXC1, forkhead box C1; miR, microRNA; OS, osteosarcoma; WT, wild-type; MT, mutant type; 3'UTR, 3' untranslated region; NC, negative control; SV40, simian vacuolating virus 40; R. luciferase, Renilla-glo luciferase; F. luciferase, firefly luciferase; HSV TK, herpes simplex virus thymidine kinase promoter; Sgf1, XhoI, PmeI and NotI, restriction sites.

Moreover, the expression of FOXC1 was significantly higher in advanced stage OS when compared with that at low stage OS. In addition, silencing of FOXC1 significantly decreased the proliferation and migration of U2OS cells, while overexpression of FOXC1 significantly promoted U2OS cell proliferation and migration. FOXC1 was identified as a direct target of miR-133b, as a reported tumor-suppressive miR in OS (19), and the expression of FOXC1 protein was negatively regulated by miR-133b in U2OS cells. Furthermore, miR-133b was significantly downregulated in OS tissues and cell lines, and levels of miR-133b were inversely correlated with the levels of FOXC1 mRNA in OS tissues.

FOXC1 is typically upregulated and serves an oncogenic role in a number of common cancers, including breast cancer (20), hepatocellular carcinoma (9), pancreatic ductal adenocarcinoma (21), non-small cell lung cancer (22) and gastric cancer (23). For instance, high expression of FOXC1 was significantly associated with poor prognosis of patients with pancreatic ductal adenocarcinoma (21), non-small cell lung cancer (22) and gastric cancer (23). A previous mechanistic study demonstrated that FOXC1 promoted the invasion of breast cancer cells by inducing the expression of matrix metalloproteinase-7 (20). In BLBC, FOXC1 enhanced the proliferation, migration and invasion of cancer cells through the activation of nuclear factor- $\kappa \mathrm{B}$ signaling (24). However, to the best of our knowledge, the expression and potential regulatory role of $\mathrm{FOXC1}$ in OS have not been previously studied. In the present study, it was observed that FOXC1 was significantly upregulated in OS tissues and cell lines. Moreover, levels of FOXC1 were higher in high stage OS than in low stage OS, suggesting that upregulation of FOXC1 may be involved in the malignant progression of OS. To further investigate the regulatory role of FOXC1 in OS, an in vitro study was conducted in $\mathrm{U} 2 \mathrm{OS}$ cells. It was observed that FOXC1 promoted the proliferation and migration of U2OS cells, suggesting that FOXC1 may serve a promotional role in the growth and metastasis of OS. Future studies are now warranted to investigate the effects of FOXC1 in OS cell growth and lung metastasis in vivo.

Several miRs have been demonstrated to directly target FOXC1, enabling them to function as tumor suppressors in different cancers $(14,25)$. For instance, it has been documented that miR-204 inhibited the migration and invasion of endometrial cancer cells by directly targeting FOXC1 (14), while miR-639 blocked tumor growth factor- $\beta$-induced EMT in human tongue cancer cells by inhibiting FOXC1 (25). Furthermore, miR-138-5p was downregulated in pancreatic cancer tissues and cell lines, and inhibited tumor cell growth in vivo by downregulating FOXC1 (26). In the present study, it was demonstrated that 
A

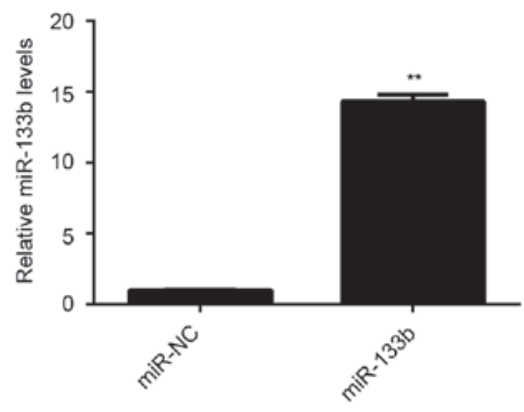

C

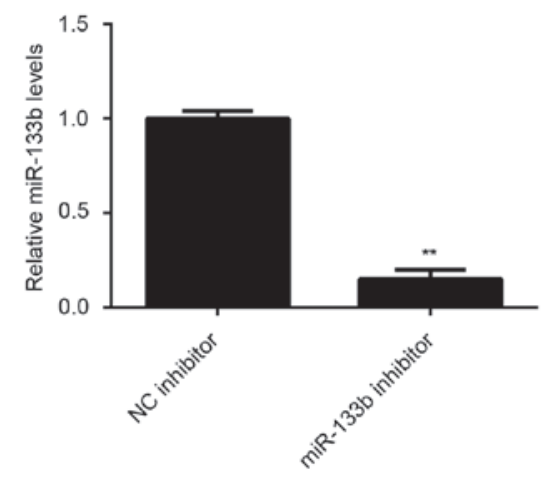

B
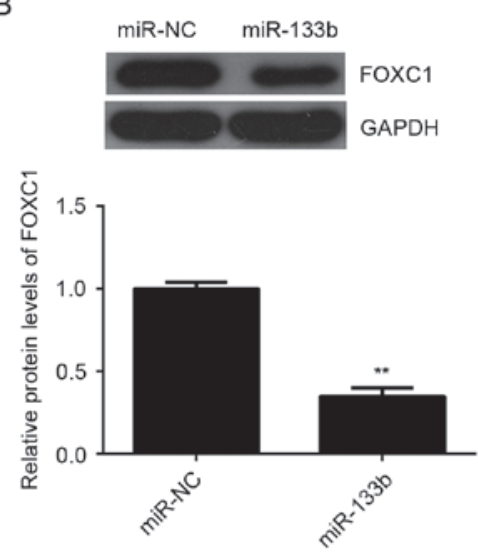

D

NC inhibitor miR-133b inhibitor
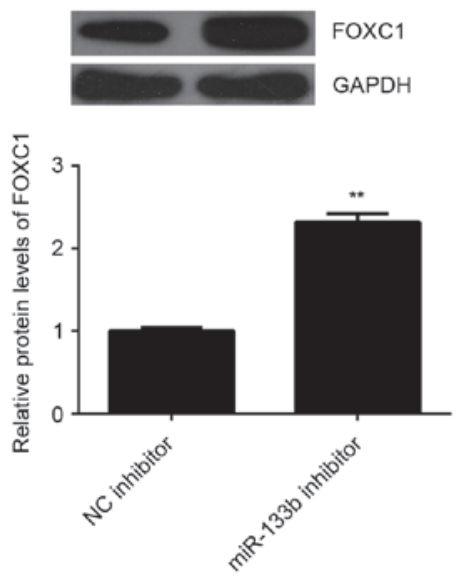

Figure 5. Targeting of FOXC1 by miR-133b in OS. U2OS cells were transfected with miR-133b or miR-NC. (A) RT-qPCR and (B) western blot analysis were performed to measure the expression of miR-133b and FOXC1 protein, respectively. U2OS cells were also transfected with miR-133b inhibitor or NC inhibitor. (C) RT-qPCR and (D) western blot analysis were performed to measure the expression of miR-133b and FOXC1 protein, respectively. ${ }^{* *} \mathrm{P}<0.01$ vs. NC. FOXC1, forkhead box C1; miR, microRNA; OS, osteosarcoma; NC, negative control; RT-qPCR, reverse transcription-quantitative polymerase chain reaction.

A

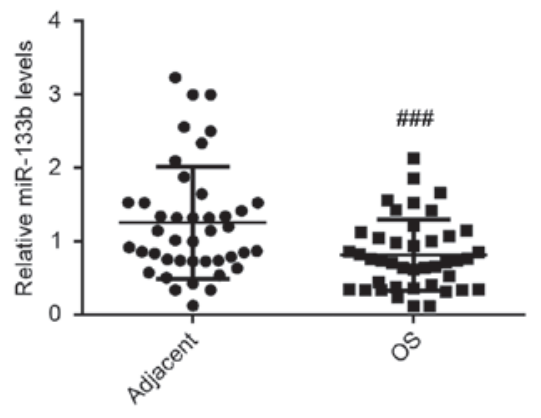

B

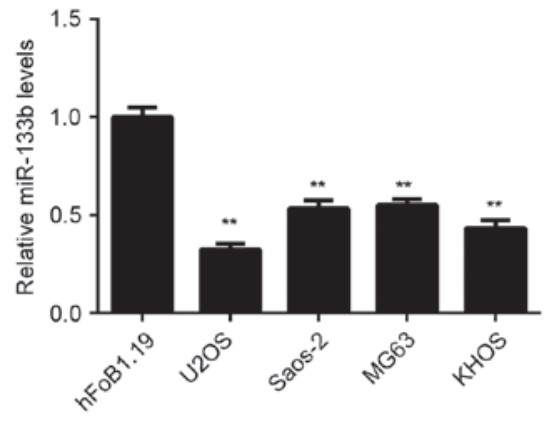

C

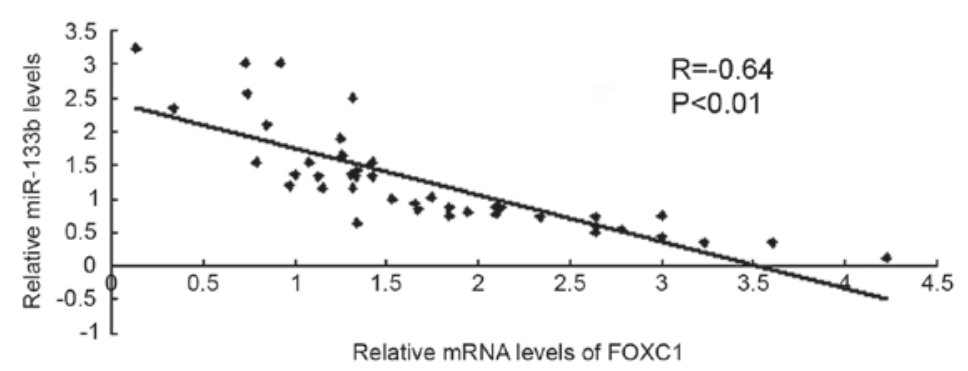

Figure 6. FOXC1 expression is inversely correlated with miR-133b expression in OS. (A) RT-qPCR was performed to assess the expression of miR-133b in OS tissues and adjacent non-tumor tissues. (B) RT-qPCR was performed to assess the expression of miR-133b in OS cell lines and normal osteoblast hFoB1.19 cells. (C) An inverse correlation was identified between the levels of miR-133b and FOXC1 mRNA in OS tissues. ${ }^{\# \# "} \mathrm{P}<0.001$ vs. adjacent tissue; ${ }^{* *} \mathrm{P}<0.01$ vs. hFoB1.19 cells. FOXC1, forkhead box C1; miR, microRNA; OS, osteosarcoma; RT-qPCR, reverse transcription-quantitative polymerase chain reaction. 
FOXC1 was a direct target of miR-133b, and miR-133b negatively regulated the expression of FOXC1 protein in OS cells. MiR-133b has previously been demonstrated to function as a tumor suppressor in OS (19). Zhang et al (19) documented that miR-133b was significantly downregulated in OS, which was associated with a high tumor grade, metastasis, recurrence and poor prognosis. Similarly, Zhao et al (27) reported that miR-133b inhibited the proliferation, migration and invasion of OS cells, and promoted their apoptosis. In the current study, it was also observed that miR-133b levels were significantly reduced in OS tissues and cell lines when compared with adjacent non-tumor tissues and normal osteoblast cells, respectively. Notably, a significant inverse correlation was identified between the expressions of miR-133b and FOXC1 in OS tissues, suggesting that decreased expression of miR-133b may contribute to the upregulation of FOXC1 in OS. Wang et al (28) recently demonstrated that miR-133 inhibited the migration and invasion of pituitary tumor cells by directly targeting FOXC1. Therefore, results of the current study provide greater insight into the miR-133b/FOXC1 axis in human cancers. Future studies should now focus on the regulatory relationship between miR-133b and FOXC1 in the growth and metastasis of OS in vivo.

In conclusion, the present results indicate that FOXC1 is directly targeted by the tumor suppressor miR-133b and serves a promotional role in the proliferation and migration of OS cells. Therefore, FOXC1 may be a novel therapeutic target in the treatment of OS. Future studies could clarify the relationship between FOXC1 and miR-133b in OS in in vivo models.

\section{References}

1. Maximov VV and Aqeilan RI: Genetic factors conferring metastasis in osteosarcoma. Future Oncol 12: 1623-1644, 2016.

2. Thompson LD: Osteosarcoma. Ear Nose Throat J 92: 288, 290 , 2013.

3. Siegel RL, Miller KD and Jemal A: Cancer statistics, 2015. CA Cancer J Clin 65: 5-29, 2015.

4. Smith RS, Zabaleta A, Kume T, Savinova OV, Kidson SH, Martin JE, Nishimura DY, Alward WL, Hogan BL and John SW: Haploinsufficiency of the transcription factors FOXC1 and FOXC2 results in aberrant ocular development. Hum Mol Genet 9: $1021-1032,2000$

5. Lehmann OJ, Ebenezer ND, Jordan T, Fox M, Ocaka L, Payne A, Leroy BP, Clark BJ, Hitchings RA, Povey S, et al: Chromosomal duplication involving the forkhead transcription factor gene FOXC1 causes iris hypoplasia and glaucoma. Am J Hum Genet 67: $1129-1135,2000$

6. Han B, Qu Y, Jin Y, Deng N, Wawrowsky K, Zhang X, Li N, Bose S, Wang Q, Sakkiah S, et al: FOXC1 activates smoothened-independent hedgehog signaling in basal-like breast cancer. Cell Rep 13: 1046-1058, 2015.

7. Huang W, Chen Z, Zhang L, Tian D, Wang D, Fan D, Wu K and Xia L: Interleukin-8 induces expression of FOXC1 to promote transactivation of CXCR1 and CCL2 in hepatocellular carcinoma cell lines and formation of metastases in mice. Gastroenterology 149: 1053-1067.e14, 2015.

8. Ray PS, Wang J, Qu Y, Sim MS, Shamonki J, Bagaria SP, Ye X, Liu B, Elashoff D, Hoon DS, et al: FOXC1 is a potential prognostic biomarker with functional significance in basal-like breast cancer. Cancer Res 70: 3870-3876, 2010.
9. Xu ZY, Ding SM, Zhou L, Xie HY, Chen KJ, Zhang W, Xing CY, Guo HJ and Zheng SS: FOXC1 contributes to microvascular invasion in primary hepatocellular carcinoma via regulating epithelial-mesenchymal transition. Int J Biol Sci 8: 1130-1141, 2012.

10. Fernebro J, Francis P, Edén P, Borg A, Panagopoulos I, Mertens F, Vallon-Christersson J, Akerman M, Rydholm A, Bauer HC, et al: Gene expression profiles relate to SS18/SSX fusion type in synovial sarcoma. Int J Cancer 118: 1165-1172, 2006.

11. Moss EG: MicroRNAs: Hidden in the genome. Curr Biol 12: R138-R140, 2002.

12. Bartel DP: MicroRNAs: Genomics, biogenesis, mechanism, and function. Cell 116: 281-297, 2004

13. Ambros V: The functions of animal microRNAs. Nature 431: 350-355, 2004

14. Chung TK, Lau TS, Cheung TH, Yim SF, Lo KW, Siu NS, Chan LK, Yu MY, Kwong J, Doran G, et al: Dysregulation of microRNA-204 mediates migration and invasion of endometrial cancer by regulating FOXC1. Int J Cancer 130: 1036-1045, 2012.

15. Li Y and Chen X: miR-4792 inhibits epithelial-mesenchymal transition and invasion in nasopharyngeal carcinoma by targeting FOXC1. Biochem Biophys Res Commun 468: 863-869, 2015.

16. Xu YY, Tian J, Hao Q and Yin LR: MicroRNA-495 downregulates FOXC1 expression to suppress cell growth and migration in endometrial cancer. Tumour Biol 37: 239-251, 2016.

17. Kundu ZS: Classification, imaging, biopsy and staging of osteosarcoma. Indian J Orthop 48: 238-246, 2014.

18. Livak KJ and Schmittgen TD: Analysis of relative gene expression data using real-time quantitative PCR and the 2(-Delta Delta C(T)) method. Methods 25: 402-408, 2001.

19. Zhang C, Yao C, Li H, Wang G and He X: Serum levels of microRNA-133b and microRNA-206 expression predict prognosis in patients with osteosarcoma. Int J Clin Exp Pathol 7: 4194-4203, 2014.

20. Sizemore ST and Keri RA: The forkhead box transcription factor FOXC1 promotes breast cancer invasion by inducing matrix metalloprotease 7 (MMP7) expression. J Biol Chem 287: 24631-24640, 2012.

21. Wang L, Gu F, Liu CY, Wang RJ, Li J and Xu JY: High level of FOXC1 expression is associated with poor prognosis in pancreatic ductal adenocarcinoma. Tumour Biol 34: 853-858, 2013.

22. Wei LX,Zhou RS, Xu HF, Wang JY and Yuan MH: High expression of FOXC1 is associated with poor clinical outcome in non-small cell lung cancer patients. Tumour Biol 34: 941-946, 2013.

23. Xu Y, Shao QS, Yao HB, Jin Y, Ma YY and Jia LH: Overexpression of FOXC1 correlates with poor prognosis in gastric cancer patients. Histopathology 64: 963-970, 2014.

24. Wang J, Ray PS, Sim MS, Zhou XZ, Lu KP, Lee AV, Lin X, Bagaria SP, Giuliano AE and Cui X: FOXC1 regulates the functions of human basal-like breast cancer cells by activating NF- $\kappa \mathrm{B}$ signaling. Oncogene 31: 4798-4802, 2012.

25. Lin Z, Sun L, Chen W, Liu B, Wang Y, Fan S, Li Y and Li J: miR-639 regulates transforming growth factor beta-induced epithelial-mesenchymal transition in human tongue cancer cells by targeting FOXC1. Cancer Sci 105: 1288-1298, 2014.

26. Yu C, Wang M, Li Z, Xiao J, Peng F, Guo X, Deng Y, Jiang J and Sun C: MicroRNA-138-5p regulates pancreatic cancer cell growth through targeting FOXC1. Cell Oncol (Dordr) 38: 173-181, 2015.

27. Zhao H, Li M, Li L, Yang X, Lan G and Zhang Y: MiR-133b is down-regulated in human osteosarcoma and inhibits osteosarcoma cells proliferation, migration, and invasion and promotes apoptosis. PLoS One 8: e83571, 2013.

28. Wang DS, Zhang HQ, Zhang B, Yuan ZB, Yu ZK, Yang T, Zhang SQ, Liu Y and Jia XX: miR-133 inhibits pituitary tumor cell migration and invasion via down-regulating $\mathrm{FOXC1}$ expression. Genet Mol Res 15, 2016 